\title{
Mechanisms of Hydrocarbon Conversion in Zeolites: A Quantum Mechanical Study
}

\author{
A. M. Rigby,* G. J. K ramer,* and R. A . van Santen $\dagger$ \\ *Shell International O il Products B. V., A msterdam, The N etherlands; and †Technical U niversity of E indhoven, E indhoven, The N etherlands
}

R eceived A ugust 5, 1996; revised D ecember 4, 1996; accepted D ecember 4, 1996

\begin{abstract}
$A b$ initio quantum chemical methods have been used to study the mechanisms for the hydrocarbon conversion in zeolites (cracking, isomerisation, alkylation, etc.). It is found that the intermediates are covalent alkoxide species and indicate that there is no energy ordering of these alkoxides according to their primary, secondary, or tertiary nature. Transition states have been located for the most important conversion reaction steps for hydrocarbons up to $\mathbf{C} 6$. The reactions are concerted and the transition states are ionic and ring-like. Due to charge delocalisation in the transition state the activation energies depend on the nature of the initial and final alkoxides. These calculations are the foundation for a new model of the hydrocarbon conversion which can better describe the influence of the zeolite as will be illustrated with calculations on acid sites with varying acid strength.

(c) 1997 A cademic Press
\end{abstract}

\section{INTRODUCTION}

Z eolites are commercially important solid acid catalysts for oil and petrochemical processes such as cracking and isomerisation of hydrocarbons (1). Given the variation in zeolite acidities, pore geometries, silicon/aluminium ratio, and in other factors such as the metal loading there is considerable flexibility in the preparation of these catalysts. $\mathrm{H}$ owever, the behaviour as a catalyst is a complex combination of the effects of reactions at the active $B$ rønsted acid site and the adsorption and diffusion of the reactants in the micropores, two effects which are experimentally difficult to disentangle. Theoretical understanding of either aspect is therefore potentially of great value in efforts to improve these catalysts.

In the literature several reaction mechanisms have been suggested, mainly on the basis of product distributions (there is much data, e.g., for cracking (2)) or isotope labelling experiments (e.g., (3)). B oth of these provide only indirect information on the reaction mechanism and no conclusive evidence for the nature of the intermediates and transition states has been reported. A Ithough there is evidence from N M R experiments (4) that alkoxides are formed at low temperatures it is not clear that these are intermediates.
The most generally successful suggestion involves positively charged carbenium ions $C R_{3}^{+}$(where $\mathrm{R}$ is an alkyl group or hydrogen) as the stable intermediates (5) formed, for instance, by protonation of an olefin by the zeolite or protolytic cracking of a paraffin. Due to charge delocalisation there is an energy ordering of the ions depending on the number of alkyl groups bound to the charged atom (primary, secondary, tertiary ions). B ased on this the mechanism can be used to rationalise some experimental data, mainly from cracking such as the faster cracking of molecules with tertiary carbon atoms and the low production of ethene (2). In an extension to this mechanism it has been suggested that protonated cyclopropane ions are involved in isomerisation (6) and possibly cracking (7) to rational ise the difference between $C_{7}$ and $C_{8}$ isomerisation and cracking. R adical reactions (8) and protolytic cracking involving carbonium ions (9) have also been suggested to rationalise the production of $\mathrm{C}_{2}$-minus hydrocarbons in cracking, particularly at high temperatures.

However, none of these mechanisms provides detailed predictions of the catalytic behaviour. In particular they do not give any description of the influence on the reactions of changes in the zeolite since the acid site ismerely a spectator counterion in most of the suggestions.

We have investigated the conversion mechanisms in more detail using ab initio quantum chemical calculations and the results are summarised in this article. A Ikoxide species covalently bound to one of the oxygen atoms in the acid site are found to be more stable than carbenium ions. E stimation in the errors made in the calculations suggest that the alkoxides are the stable intermediates for conversion reactions in real zeolites as was first suggested by K azansky and Senchenya (10). The primary, secondary, or tertiary nature of these alkoxides is found to have little influence on their stability in contrast to carbenium ions.

We have also located the transition states for the cataIytically important reaction steps. M ost of them are found to be ionic, with the hydrocarbon fragment being positively charged. They therefore resemble carbenium or carbonium ions. A $n$ ordering is found depending on the primary, secondary, or tertiary nature of the initial and final alkoxides. 
This suggests that the success of the carbenium ion model's predictions of preferred reaction pathways is due to an ordering of the barriers to reaction rather than the relative stability of the intermediates.

B eyond reproducing the prediction of the preferred pathways (albeit for different reasons), the new model for the conversion mechanisms suggested by the calculations but has important differences and goes considerably further. In particular the zeolite acid site is not a passive counterion in the reactions but plays an intimate role in the intermediates and the transition states. It is now possible to investigate how variations in the zeolite would influence the reaction mechanisms. In particular the semiquantitative data from the calculations can be used to investigate some of the issues important for catalysis such as the effect of differences in the acid strength of the zeolite sites, differences between hydrocarbons, or estimates of the relative activation energies of different steps.

\section{METHODS: CHOICE OF MODEL AND COMPUTATIONAL DETAILS}

A II calculations were performed using the G A M E SS-U K ab initio code (11). The geometries of all states were fully optimised (within the symmetry constraints described below) at the $\mathrm{H}$ artree-Fock level using the $3-21 \mathrm{~g}$ basis set. Single-point calculations using the $6-31 g^{*}$ basis set and second-order M P2 perturbation theory correlation corrections were performed at these geometries. These singlepoint results are found to give activation energies close to those for structures fully optimised with the 6-31g* basis set, despite relatively large changes in the energies of individual species. A s an example the difference in the single-point and fully optimised activation energies for cyclopropane ring opening and closure is only 1.0 and $1.2 \mathrm{kcal} / \mathrm{mol}$. For ethene chemisorption the difference is 4.3 and $3.9 \mathrm{kcal} / \mathrm{mol}$.

Transition states were located using the Gamess saddle point search routine based on an internal coordinate z-matrix. The initial z-matrix was defined using typical bond lengths and angles from previous experience. The z-matrix was chosen to concentrate the reaction coordinate in as few as possible internal coordinates and a saddle point sought for these variables (restarting from the best point if necessary). The remaining variables were added in stages by energy minimisation followed by saddle point searches.

It should be noted that there are two slightly different versions of the 6-31g* basis set for silicon. Here the G amess (UK) default (12) was used but in other codes such as Gaussian the version in (13) is used. This has negligible influence on the quantities of interest (such as activation energies) but should be taken into account when comparing total energies from different codes.

The active Brønsted site was modelled by the cluster shown in Fig. 1 with the central aluminium atom linked

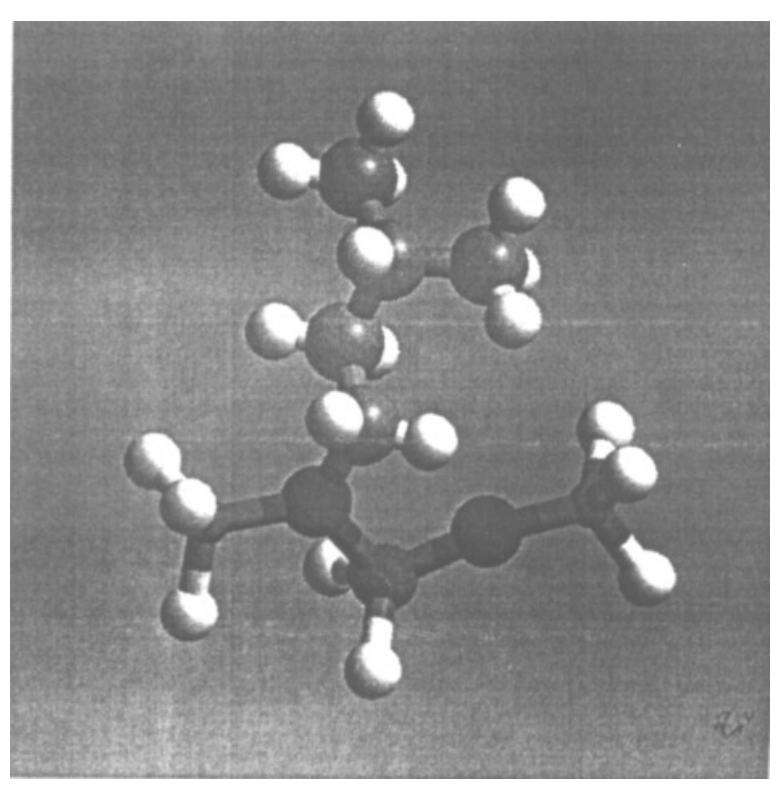

FIGURE 1

to two silicon atoms via bridging oxygen atoms. A s will be seen this is the minimal cluster for realistic modelling of the reactions, as two oxygen atoms are involved. The cluster is terminated by hydrogens rather than hydroxyl groups since Curtiss et al. (14) showed that this is a better model for the rest of the zeolite. We have shown in the past that such a cluster can be used to study reactions in zeolites $(15,16)$. The cluster does not allow the calculation of the full binding energies of the hydrocarbon. H owever, since the effect of the remainder of the lattice will be similar for all the states considered, the relative activation energies deduced will be relatively accurate and these are the quantities of interest for catalysis.

A s was shown in $(15,16)$ the acidity of this cluster can be changed by constraining the terminal $\mathrm{Si}-\mathrm{H}$ distances. The variation in the acidity can be measured by the variation in the calculated cluster deprotonation energy or its ammonia chemisorption energy which vary by 20.2 and $5.3 \mathrm{kcal} / \mathrm{mol}$, respectively, for a variation of the bond length from 1.3 and $1.7 A^{\circ}$. U sing these constrained clusters the influence of acidity variation on the reactions can be investigated.

A s will be seen, the majority of the transition states resemble carbenium ions adsorbed to the acid site. The energy surface for displacements of the ion is relatively flat, making location of transition states difficult. Therefore, for computational convenience a symmetry plane (in the plane of the acid site) was imposed on most of the present calculations. F urther calculations (17) indicate that the effect on the quantities of interest (e.g., relative activation energies of different reaction steps) is limited although the geometries of some states are significantly nonplanar. For example, removal of the symmetry constraint for the protolytic 
cracking of propane and isobutane alters the calculated activation energy by around $10 \mathrm{kcal} / \mathrm{mol}$ (see below). Note that the "fully optimised" values quoted in this paper still have the $\mathrm{Si}-\mathrm{O}-\mathrm{A} \mathrm{I-O}-\mathrm{Si}$ atoms of the cluster constrained to lie in a plane to mimic the effects of the lattice and to avoid two soft modes which are not present in the real zeolite. For those states with nonsymmetrical hydrocarbons (such as the methyl shift in pentene) the $\mathrm{A}-\mathrm{O}-\mathrm{C}-\mathrm{C}-\mathrm{C}-\mathrm{O}$ ring at the centre of the transition state was confined to the plane of the acid site. The transition states found with such constraints need not be the true transition states but later work (17) has found that releasing the constraints has little effect on the nature of the transition state and a limited effect on the relative activation energies, although the detailed geometry of the transition state can be significantly different. For hydride transfer the energy surface is particularly shallow and true transition states are difficult to locate. Hence, for convenience, we quote the energies of the $C_{2 v}$ symmetry states with the transferring hydrogen halfway between the two hydrocarbons. These are energy minima but are close in energy to the transition states. For i-butyl/i-butyl there is a $0.3 \mathrm{kcal} / \mathrm{mol}$ difference between this state and the state in which the $\mathrm{C}-\mathrm{H}-\mathrm{C}$ axis is perpendicular to the plane of the acid site which is a transition state for the transfer (as was suggested by K azansky et al. (18) using a smaller cluster).

\section{RESULTS: INTERMEDIATES}

The first step in investigating a reaction mechanism is the identification of the stable intermediates. It is found that alkoxide species covalently bound to one of the bridging oxygens of the acid site (illustrated in Fig. 1) are stable in the calculations but carbenium ions are not. A Ithough they are much higher in energy, local energy minima (i.e., metastable states) can be found for tertiary carbenium ionslarger than isobutane, if proton transfer to the site is prevented by rotating the tail of the hydrocarbon towards the cluster. Examples of the calculated relative energies for these ionic states and the alkoxide where the tail of the hydrocarbon has also been rotated are shown in Table 1. This gives an estimate of the energy difference between alkoxides and tertiary carbenium ion states of around $20 \mathrm{kcal} / \mathrm{mol}$. We thus conclude that the stable intermediates in the conversion are alkoxide species as suggested by K azansky (10).

Further, we have calculated the relative energies of the primary, secondary, and tertiary alkoxides of the same hydrocarbon. In particular the calculated relative energies of the four different alkoxides of methylbutane with no symmetry constraints are shown in Table 1. The alkoxides have similar energies and there is no particular ordering according to their primary, secondary, and tertiary nature. This contrasts with calculations of free carbenium ion energies where there is a clear ordering with an energy difference of around $15 \mathrm{kcal} / \mathrm{mol}$ between primary and secondary and
TABLE 1

Relative Energies $(\mathrm{kcal} / \mathrm{mol})$ of the Free Site and $i$-hex-2-ene versus the $C$ arbenium I on State, the Lowest E nergy Alkoxide, and the Alkoxide with the Same Constraints on Its $\mathrm{H}$ ydrocarbon Tail as the Ion

\begin{tabular}{|c|c|c|c|}
\hline Species & $3-21 \mathrm{~g} / \mathrm{scf}$ & $\begin{array}{c}6-31 g^{*} / \mathrm{scf} / / \\
3-21 \mathrm{~g} / \mathrm{scf}\end{array}$ & $\begin{array}{c}\text { 6-31g*/M P2// } \\
3-21 \mathrm{~g} / \mathrm{scf}\end{array}$ \\
\hline Free site and i-hexene & 0.0 & 0.0 & 0.0 \\
\hline Tertiary carbenium ion & +39.9 & +30.2 & +27.9 \\
\hline $\begin{array}{l}\text { Lowest energy alkoxide } \\
\text { (tertiary) }\end{array}$ & -7.8 & -2.1 & -14.7 \\
\hline $\begin{array}{l}\text { Constrained alkoxide } \\
\text { (tertiary) }\end{array}$ & +17.6 & +20.8 & +7.0 \\
\hline A mmonia chemisorption energy & 26.79 & 12.38 & 26.71 \\
\hline $\begin{array}{l}\text { 1-methyl-butyl alkoxide } \\
\text { (primary) }\end{array}$ & -0.12 & -2.48 & 4.14 \\
\hline $\begin{array}{l}\text { 2-methyl-butyl alkoxide } \\
\text { (tertiary) }\end{array}$ & 0.0 & 0.0 & 0.0 \\
\hline $\begin{array}{l}\text { 3-methyl-butyl alkoxide } \\
\text { (secondary) }\end{array}$ & -1.54 & -2.59 & 0.05 \\
\hline $\begin{array}{l}\text { 4-methyl-butyl alkoxide } \\
\text { (primary) }\end{array}$ & -0.83 & -2.07 & 3.46 \\
\hline
\end{tabular}

Note. A mmonia chemisorption energies and relative energies $(\mathrm{kcal} / \mathrm{mol})$ of methylbutane alkoxides as a function of their binding position.

between secondary and tertiary ions. Since such an ordering of the energies of the intermediates is the basis for the predictions of the carbenium ion model there must be another explanation of the success of these predictions. This will be discussed below.

\subsection{Charge L ocalisation and Polarisation E ffects}

There is a potential problem in using relatively small clusters to model the acid site to compare the energies of ionic and covalent states which should be addressed. The stabilisations of ionic states by spreading the negative charge to the remainder of the lattice and by polarising it are neglected. In the absence of a positive counterion these effects can be large, as is shown by calculations of the deprotonation energy (19) which only converge for large clusters. $\mathrm{H}$ owever, the states of interest to catalysis do have a positive counterion close to the deprotonated acid site (for instance, a charged hydrocarbon). The field of thision tends to reduce the spreading of the negative charge and the polarisation of the lattice, reducing the errors in the calculations. This is illustrated by comparing calculated and experimental ammonia chemisorption energies. For bidentate adsorption of the ammonium ions, the calculated chemisorption energies are close to the experimental values $(20-25 \mathrm{kcal} / \mathrm{mol}$ depending on the zeolite (20)) as is shown in Table 1. This suggests that any error is less than about $10 \mathrm{kcal} / \mathrm{mol}$ and that even tertiary carbenium ions are less stable than alkoxides in real zeolites as well as in our clusters. The error in using a small 
cluster is then in the underestimation of the acidity and this can be corrected by adjusting the acidity of the cluster by constraints of $\mathrm{Si}-\mathrm{H}$ bond distance as described below. It should be noted that these calculations were performed to estimate the errors for the basis sets and zeolite cluster used here and not to accurately model ammonia chemisorption which has already been addressed (20).

\section{RESULTS: TRANSITION STATES}

$\mathrm{H}$ aving identified the intermediates, the transition states for the conversion steps must be located. In addition to our work on the $\mathrm{D} / \mathrm{H}$ exchange in methane $(15,16)$, the transition states for chemisorption of olefins forming an alkoxide have been studied by K azansky (ethene (21)) and Corma (propene and isobutene (22)). Protolytic cracking of small paraffins has been studied by various groups $(18,23-26)$ as has protolytic dehydrogenation $(18,26-28)$. Some work has also been done on hydride transfer $(18,26)$. D ehydration of methanol has also been studied $(29,30)$.

However, many of the conversion steps important for the majority of the commercial processes have not been published. The important steps and transition states are:

- O lefin adsorption/desorption to form an alkoxide via protonation of the other end of the double bond which will be the mechanism for olefins to enter or leave the conversion reactions.

- $\beta$-Scission/oligomerisation in which an alkoxide cracks to give a smaller alkoxide and an olefin. This will be involved in cracking at moderate temperatures and (in the reverse direction) oligomerisation and alkylation reactions.

- Hydride transfer in which a hydrogen atom is transferred from an incoming paraffin to an alkoxide, transforming the alkoxide into a paraffin and the initial paraffin into an alkoxide. This will be involved in bringing a new paraffin into cracking reactions in the absence of a hydrogenation function and a possible equilibrium between olefins and alkoxides.

- Internal hydride methyl and alkyl shifts in which the position of the alkoxide bond and the hydride or methyl group is exchanged. This is involved in the isomerisation of paraffins and olefins and in alkylation (32) and cracking.

- Ring opening/closure to form a substituted cyclopropane. This is the alkoxide version of the protonated cyclopropane mechanism originally suggested by B rouwer and $\mathrm{H}$ oogeveen (6).

- Protolytic cracking: This is the direct cracking of a paraffin via protonation by an acid site. It may be involved in cracking at higher temperatures and in the initiation of alkane cracking. It is an analogue of the carbonium ion mechanism (9).

Transition states have been located for all these reaction steps (with the partial exception of hydride transfer as discussed in the Computational Details section). Figure 2
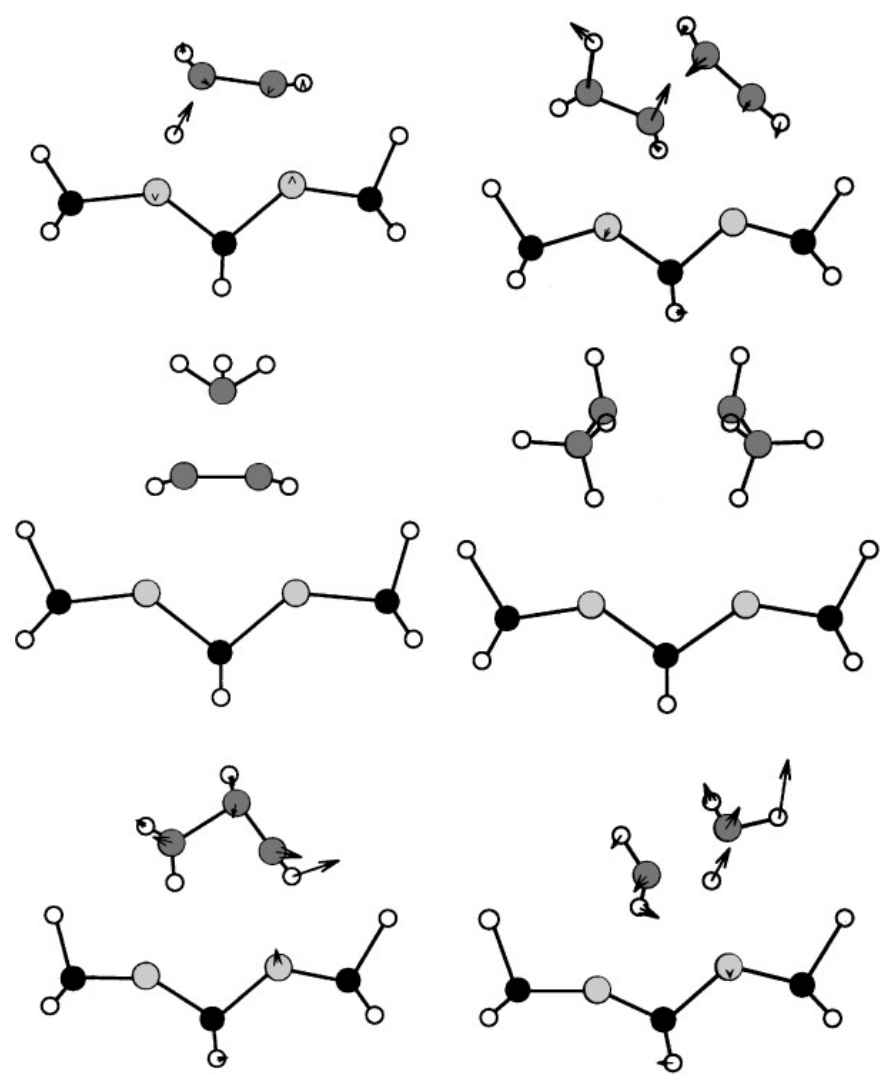

FIGURE 2

shows the structure and reaction coordinate for examples with small hydrocarbons (for clarity). Calculated activation energies in various computational approximations are shown in Table 2. Total energies are shown in Table 3 to aid any attempts to reproduce the results. These values will be compared with experiment after a discussion of the general features of the transition states. H owever, we note at this point that the values appear large due to the combined effects of the small hydrocarbons involved, the underestimation by the cluster of the acidity of the real site, due to the charging effects mentioned in the Computational D etails section, and because the values quoted are relative to the alkoxide species which is usually significantly more stable than the reactant molecules. For low alkoxide coverage (high temperatures) the observed activation energy will be lower by this difference in energies.

The transition states are all found to involve five or six ring structures formed from the $\mathrm{O}-\mathrm{Al}-\mathrm{O}$ atoms of the cluster and two or three carbon or hydrogen atoms of the hydrocarbon. Given the results on the intermediates in the reaction, this is natural since the initial and final states must have a species covalently bound to one of the oxygens of the site, being either an alkoxide or an acid proton. Steric considerations prohibit the use of the same oxygen leading to ring-like structures. 
TABLE 2

Activation E nergies (kcal/mol) of the Various R eaction Steps Relative to the Initial Alkoxide and Physisorbed Reactant, if Any

6-31g*/scf// 6-31g*/M P2//

R eaction

$3-21 \mathrm{~g} / \mathrm{scf} \quad 3-21 \mathrm{~g} / \mathrm{scf} \quad 3-21 \mathrm{~g} / \mathrm{scf}$

E thene chemisorption

Forward

R everse

30

42

39

51

23

i-B utene chemisorption (primary)

Forward

R everse

37

48

i-B utene chemisorption (tertiary)

Forward

R everse

$H$ ydrogen shift in ethyl alkoxide Forward

$\mathrm{H}$ ydrogen shift in $\mathrm{n}$-butyl alkoxide Forward

M ethyl shift in propyl alkoxide Forward

$M$ ethyl shift in i-pentyl alkoxide Forward

$\beta$-Scission of $\mathrm{n}$-butyl alkoxide

Forward

R everse

$\beta$-Scission of isohexyl alkoxide

Forward

R everse

$\mathrm{H}$ ydride transfer methanemethane

Forward

Hydride transfer propanepropane

Forward

H ydride transfer isobutaneisobutane

Forward

Cylopropane ring closure

Forward

R everse

Protolytic cracking of ethane

Forward

R everse

Protolytic cracking of propane

Forward

R everse

Protolytic cracking of propane

Forward

R everse

Protolytic cracking of $n$-butane (to $\mathrm{CH}_{4}$ and $\mathrm{C}_{3} \mathrm{H}_{6}$ )

Forward

R everse

Protolytic cracking of n-butane (to $\mathrm{CH}_{4}$ and $\mathrm{C}_{3} \mathrm{H}_{6}$ )

Forward

R everse

Protolytic cracking of i-butane

Forward

R everse

Protolytic cracking of i-butane

Forward

R everse
28

39

77

69

82

72

75

57

68

48

93

73

58

82

48

69

46

77

49

95

89

92

78

78

70

77

82

80

68

89

84

77

81

80

67

84

83

68

81

74

60

The transition states are also found to be ionic with the exception of the $\mathrm{D} / \mathrm{H}$ exchange and olefin chemisorption. The positive Mulliken charge on the hydrocarbon fragment is typically $+0.8 \mathrm{e}$. $\mathrm{D} / \mathrm{H}$ exchange is found to be covalent ( $M$ ulliken charge of 0.47 e) and the olefin chemisorption is an intermediate case ( $\mathrm{M}$ ulliken charge $+0.54 \mathrm{e}$ ). This difference in nature is also reflected in the calculated distances between the acid site and the hydrocarbon. For instance, the

\section{TABLE 3}

\section{Selected Total Energies (Atomic U nits) at the Level U sed for Optimisation (3-21g/scf)}

N ote. A Iso energies of tertiary isohexyl carbenium ion relative to alkoxide. A II reactions with $\mathrm{Si}-\mathrm{H}$ distances set to $1.5 \AA^{\circ}$ with planar symmetry apart from fully optimised. 
distances from the site's oxygen atoms to the transferring hydrogen for $D / H$ exchange is $1.157 A^{\circ}$, in olefin chemisorption it is $1.219 \AA^{\circ}$, and in protolytic cracking of ethane it is $1.965 \AA^{\circ}$. These differences will be reflected in the acid strength dependence of the activation energies described below.

The hydrocarbon fragments of the transition states therefore resemble the transition states for the corresponding reactions in free gas-phase carbenium ions (although this separation is not complete as will be shown in the section on the influence of acidity differences). The positive charge is concentrated on the carbon atoms forming the initial and final alkoxide species (if any) and the energy of carbenium ions depends on their primary, secondary, or tertiary nature. $\mathrm{H}$ ence the energy of the transition state and the activation energy depend on whether the initial and final alkoxides are primary, secondary, or tertiary as can be seen in the values quoted for the $\beta$-scission, olefin chemisorption, hydride transfer, and hydride and methyl shifts as can be seen from Table 3. This suggests that the broad predictions of the carbenium ion model are successful due to an ordering of the energies of the transition states rather than an ordering of the intermediates. Thus, for instance, pathways involving tertiary carbenium ions or alkoxides are favoured over those involving primaries because the reaction barriers are lower rather than because the intermediates are more stable.

The calculations suggest a new picture of the conversion mechanisms which reproduces the broad predictions of the carbenium ion model (albeit for different reasons) but has important differences and goes considerably further. In particular the zeolite acid site is not a passive counterion in the reactions but plays an intimate role in the intermediates and the transition states and it is easier to see how variations in its properties would influence the reaction mechanisms. The structure and energy of a transition state is determined by the interplay of the steric interactions, the electrostatic forces between the charged site and the hydrocarbon fragments and the electronic structure of the partially formed and broken bonds. In hydride transfer, for instance, the hydrocarbon fragments are relatively bulky and steric considerations force them to be a relatively large distance from the acid site, increasing the charge separation energy. This gives the hydride transfer step a high activation energy in the calculations even though a negligible activation energy is calculated for hydride transfer for free carbenium ions (32). This activation energy will vary with the degree of steric hindrance of the acid site involved. This is important, for instance, in alkylation (32) where the high barrier gives time for isomerisation of product alkoxides and favours the production of only those paraffins with tertiary carbon atoms.

F urther the calculations also give useful semiquantitative data, whereas the carbenium ion model is mainly used to predict favoured pathways. In particular the calculations yield estimates for the relative activation energies of the different conversion steps. M ore importantly for catalyst research, the variations in the activation energies with changes in the hydrocarbon (such as the degree of branching) or the acid strength of the zeolite acid site (described below) can be predicted. I is expected that these values will be significantly more accurate than the values calculated for given activation energies since there will be a large cancellation of errors.

Comparison of the calculated data with experiment is difficult for most of the catalytically important reaction steps since usually several of them are taking place at the same time and it is difficult to measure them independently. O ne exception is the protolytic cracking of paraffins. Corma et al. (33) finds an activation energy for isobutane cracking in U SY of $51.6-53.6 \mathrm{kcal} / \mathrm{mol}$ (after correction for the effects of physisorption energies using the values in (34)). $\mathrm{N}$ arbeshuber et al. (35) obtains $47.3 \mathrm{kcal} / \mathrm{mol}$ for propane and $47.1 \mathrm{kcal} / \mathrm{mol}$ for $\mathrm{n}$-butane in $\mathrm{H}-\mathrm{ZSM}-5$. The calculated values for propane, $n$-butane, and isobutane with and without symmetry constraints are given in Table 2 . It can be seen that the effect of removal of the symmetry constraint is around $10 \mathrm{kcal} / \mathrm{mol}$. The values with no imposed symmetry are 6 and around $20 \mathrm{kcal} / \mathrm{mol}$ higher than the Corma et al. and $\mathrm{N}$ arbeshuber et al. values, respectively. A round $5-10 \mathrm{kcal} / \mathrm{mol}$ of this difference can be ascribed to the underestimation of the acidity of the real zeolite by the small cluster discussed above. The remaining $0-10 \mathrm{kcal} / \mathrm{mol}$ is likely to be due to deficiencies in the basis set or the correlation corrections used. M ost of these errors are expected to be consistent for different reactions.

\section{RESULTS: INFLUENCE OF DIFFERENCES IN THE ACID STRENGTH ON THE ACTIVATION ENERGIES}

Of particular interest for understanding differences in catalytic activity is the influence of the changes in the catalyst on the reaction barriers. The zeolite plays an intimate role in all the intermediates and transition states discussed above, whereas it is only a spectator counterion in the carbenium ion mechanisms once it has protonated the hydrocarbon. It is therefore much easier to understand the effects on the reactions of changes in the zeolite and it is possible to make predictions of some of the influences based on the calculations. In particular the effects on the reactions of differences in the strength of the acid site can be investigated now and the effects of steric hindrance can be investigated when larger zeolite clusters can be considered.

A s described in our work on $D / H$ exchange $(15,16)$, the acid strength of the cluster used in these calculations can be varied by constraining terminal $\mathrm{Si}-\mathrm{H}$ bonds to have various lengths. This strength variation can be measured by calculations of the ammonia chemisorption energy or the 
deprotonation energy which vary by 5.2 and $20.2 \mathrm{kcal} / \mathrm{mol}$, respectively, for a variation of the bond length from 1.3 to $1.7 \AA$. This variation in the deprotonation energy approximately spans the range of acidity variations that was calculated to occur in zeolites (36).

D espite the comments above about the absolute value of the deprotonation energies calculated for small clusters, it does vary linearly with the ammonia chemisorption energy and so either can be used as an estimate of acidity variation.

In real sites the two oxygen atoms involved in the reaction can have different acidities due to different geometric constraints or different local Si/A I distributions. This can be modelled in the cluster if the $\mathrm{Si}-\mathrm{H}$ bonds at the two ends of the cluster are set to different values. Variation of the bonds at one end of the cluster has around three times as much influence on the acid strength of the oxygen atom at that side of the cluster as on the second oxygen. The influence of both the absolute acid strength and the differences in the strengths of the two oxygens have been investigated.

The variation with the absolute acid strength (i.e., when the acidity of the two oxygens is varied jointly) of the activation energies of the reaction steps of interest for hydrocarbon conversion is shown in Fig. 3. There is little effect on the activation energies for $\mathrm{D} / \mathrm{H}$ exchange in methane and for olefin chemisorption which have covalent transition states. The energies of the alkoxides relative to the acid site is also very little changed. $\mathrm{H}$ owever, there is a strong influence on the energies of the other steps which have ionic transition states. It can be seen from Fig. 3 that all of these activa- tion energies are strongly reduced with increasing acidity. M ost of the activation energies are influenced to a similar extent so the variation in the relative rates for these reaction steps is much less than the change in overall rate but it can still be significant catalytically. For the range of acidities shown in Fig. 3 the overall reaction rate would increase by a factor of around $10^{4}$ at typical reaction temperatures of $500 \mathrm{~K}$ but the relative rates will be changed by a factor of around 20. Since the chemisorption of olefins is much less sensitive to the acidity, the relative rates of this and other steps will vary greatly and this may be significant for processes with hydrogenating functions where the relative rates of olefin chemisorption and other reactions may be important.

The distinct differences between the ionic and covalent transition states can be readily understood since the B rønsted acid strength is essentially a measure of the energy difference between a covalent and an ionic state (the acid-base pair before and after proton transfer). Hence variations in acidity do not greatly influence the relative stabilities of covalent states (the acid site, the alkoxide, and the transition state for olefin chemisorption) but do affect the energy differences between one of these states (the alkoxide) and ionic transition states. However, this relation between the ionic nature of the transition state and the sensitivity to acidity cannot be taken too far as is shown by Fig. 4 where the sensitivity to acidity is plotted against the $M$ ulliken and $L$ owdin charges on the hydrocarbon fragment in the transition state. The difference between ionic and

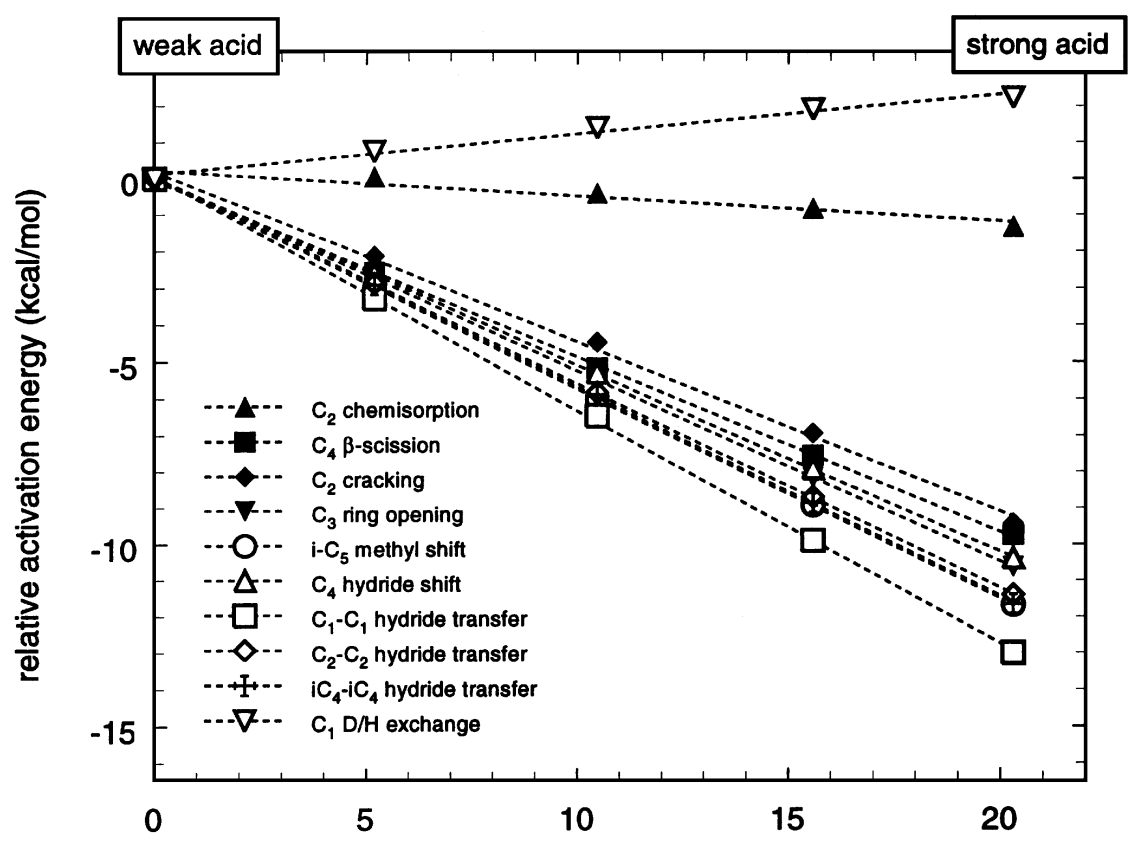

relative proton affinity $(\mathrm{kcal} / \mathrm{mol})$

FIGURE 3 


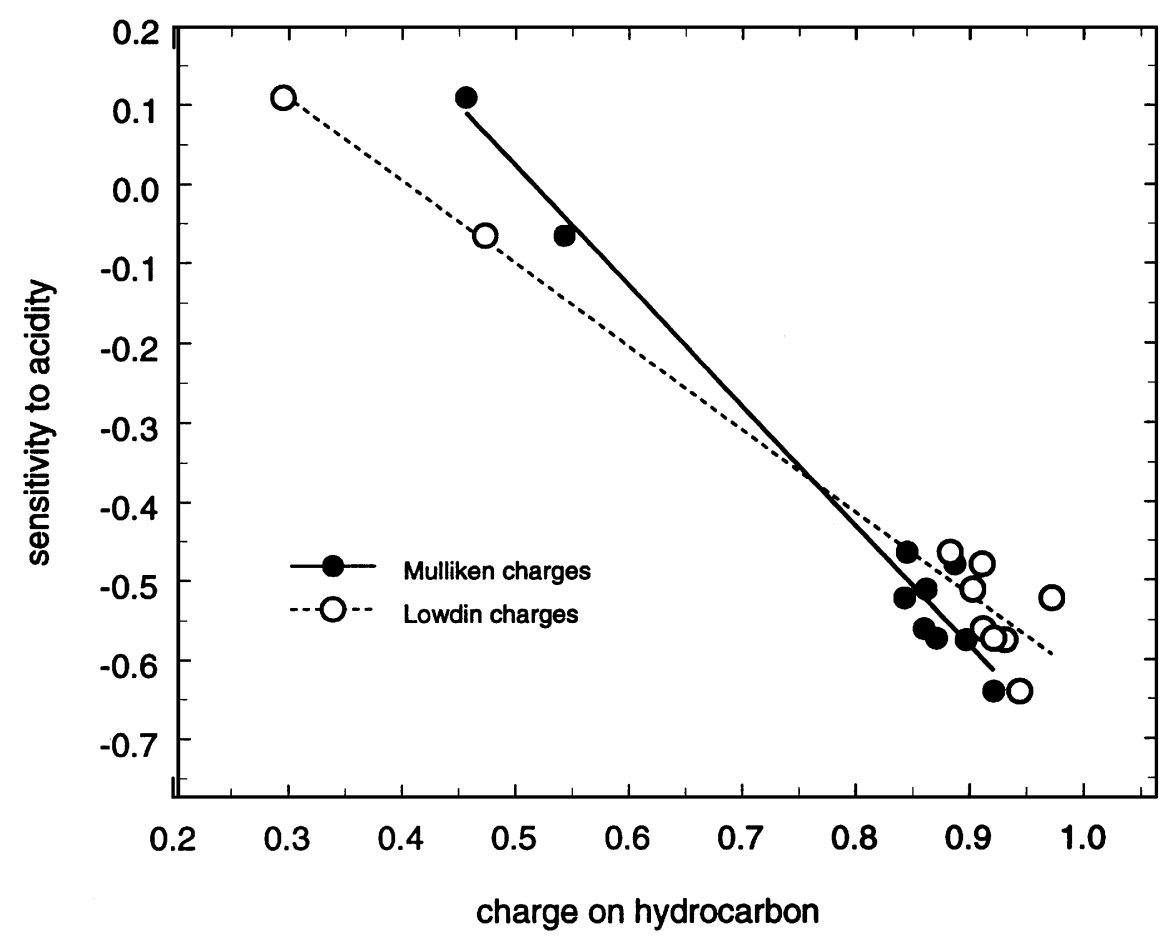

FIGURE 4

covalent states is well described but the detailed differences between ionic transition states cannot be deduced.

The variation of the activation energies with the difference in the strength of the two oxygen atoms in the acid site is shown in Fig. 5. The values shown are the deviations in the calculated activation energies from the deprotonation energy of the oxygen bonded to the initial acid proton or alkoxide species (i.e., the deviation from the line in Fig. 4). It can be seen that the basicity of the second oxygen has a significant impact on the activation energy.

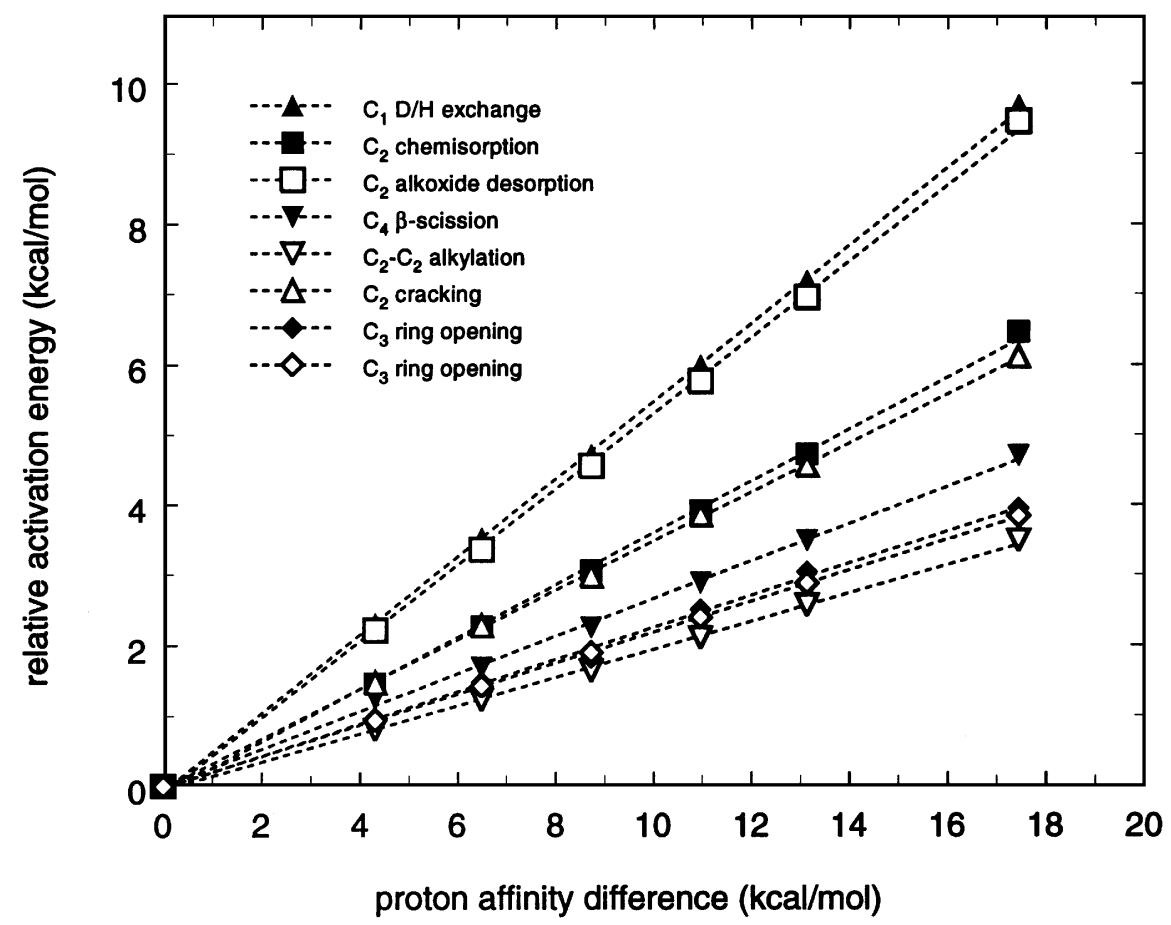

FIGURE 5 
It can be seen that there is again a clear difference between the covalent reaction steps ( $D / H$ exchange and olefin chemisorption) and the other steps which are more ionic. This is understandable since in $\mathrm{D} / \mathrm{H}$ exchange and olefin chemisorption there is a balance between the forming and breaking bonds $(15,16)$. H owever, the results show that the other states are also not completely ionic since they also have such an effect. Since the variation shown can also be significant chemically this suggests that the acid strength of more than one of the oxygen atoms of the site should be taken into account in attempts to correlate the activity against acidity data.

\section{CONCLUSIONS}

A b initio calculations indicate that the stable intermediates in hydrocarbon conversion reactions in zeolites are covalent alkoxide species with no energy ordering according to their primary, secondary, or tertiary nature in contrast to the carbenium ion model of the conversion reactions. Examples of the important transition states have been located for hydrocarbons up to C 6 (with the partial exception of hydride transfer). The reactions are found to be concerted with ring-like transition states and different oxygen atoms involved in the initial and final alkoxide or acidic $\mathrm{O}-\mathrm{H}$ bonds.

The hydrocarbon portion of most of these states is found to be positively charged and resembles the corresponding transition state in free carbenium ions. Exceptions are the chemisorption of olefins and the $\mathrm{D} / \mathrm{H}$ exchange in methane which are more covalent in character and show little charge accumulation on the hydrocarbon fragment. The activation energy for the ionic steps depends on the number of alkyl groups bound to the carbon atoms which form the initial and final alkoxide species (if any) since the charge is concentrated on these atoms and in this respect they resemble carbenium ions. Hence the success of the carbenium ion model predictions of preferred reaction pathways is believed to be due to variation in the reaction barriers with the primary, secondary, or tertiary nature of the alkoxide intermediate rather than an energy ordering of the intermediates themselves.

B eyond this picture of the general features of the conversion mechanisms the data from the calculations can be used to investigate the influence of changes in zeolite on the conversion reactions. Since the zeolite is seen to play an intimate role in the intermediates and the transition states in the conversion it is easier to see how changes in the zeolite will affect the reactions. In particular calculations on the variation of the activation energies with acid strength show that the rates of the majority of the conversion steps vary similarly with the acid strength. The exception is the olefin adsorption which varies more weakly. H ence the relative rates of most of the steps are affected much less than the overall rate but this is still significant for the catalysis. Varying the difference in the acidities of the two oxygen atoms involved in the reaction has more effect on the olefin chemisorption than the other reaction steps but these are also affected showing that the hydrocarbon is not completely separated from the acid site. This also implies that the acidity of both oxygen atoms should be taken into account in studies of the reactions.

\section{REFERENCES}

1. M axwell, I. E ., and Stork, W. H . J., in "I Introduction to Z eolite Science and Practice" (H. van Bekkum et al., Eds.), pp. 571-630. Elsevier, A msterdam, 1991.

2. Krannila, H., Haag, W. O., and Gates, B. C., J. Catal. 135, 115 (1992).

3. A suquo, R. A ., E der-M irth, G., and Lercher, J. A ., J. Catal. 155, 376 (1995).

4. H aw, F. J., R ichardson, B. R., O shiro, I. S., L azo, N. D., and Speed, J. A ., J. A m. Chem. Soc. 111, 2052-2058 (1989).

5. Martens, J. A ., and Jacobs, P. A ., in "Theoretical A spects of $\mathrm{Het-}$ erogeneous Catalysis" (J. B. M offat, E d.), Chapter 2. Van N ostrandR einhold, N ew Y ork, 1990.

6. Brouwer, D. M., and H oogeveen, H., Prog. Phys. Org. Chem. 9, 179 (1972).

7. Sie, S. T., Ind. Eng. Chem. D es. 31, 1881 (1992).

8. Corma, A ., Stud. Surf. Sci. Catal. 49, 49 (1989).

9. Haag, W. O., and Dessau, R. M., in "Proceeding, 8th International Congress on Catalysis, Berlin, 1984," Vol. II, p. 305. Dechema, Frankfurt-an-M ain, 1984.

10. Kazansky, V. B., and Senchenya, I. N., J. Catal. 119, 108 (1989).

11. Guest, M. F., Fantuicci, P., H arrison, R. J., Kendrick, J., van Lenthe, J. H., Schoeffel, K., and Sherwood, P., "GAMESS-UK U ser's Guide and Reference Manual 1: Revision C.O." Computing for Science (CFS) Ltd., D aresbury Laboratory, D aresbury, UK, 1993.

12. G ordon, M. S., Chem. Phys. L ett. 76, 163 (1980).

13. (a) Gordon, M. S., B inkley, J. S., Pople, J. A ., Pietro, W. J., and H ehre, W. J., J. A m. Chem. Soc. 104, 2979 (1982); (b) Fransi, M. M., Pietro, W. J., H ehre, W. J., B inkley, J. S., G ordon, M. S., de Frees, D. J., and Pople, J. A ., J. Chem. Phys. 77, 3654 (1982).

14. B rand, H. V., Curtiss, L. A ., and Iton, L. E., J. Phys. Chem. 19, 6435 (1991).

15. K ramer, G. J., van Santen, R. A ., E meis, C. A ., and Nowak, A . K., Nature 363, 529 (1993).

16. K ramer, G. J., and van Santen, R. A ., J. A m. Chem. Soc. 117, 1766 (1995).

17. Rigby, A . M ., and Frash, M . V., manuscript in preparation.

18. K azansky, V. B., Frash, M. V., and van Santen, R . A ., A ppl. Catal., in press.

19. B randt, H. V., Curtiss, L. A ., and Iton, L. E., J. Phys. Chem. 97, 12773 (1993).

20. Experimental data from microcalorimetry and ammonia TPD from several sources as quoted in Teunissen, E. H., van Santen, R. A ., Jansen, A. P. J., and van D uijneveldt, F. B., J. Phys. Chem. 97, 203 (1993).

21. Senchenya, I. N., and K azansky, V. B., Catal. L ett. 8, 317 (1991).

22. Viruela-M artin, P., Zicovich-Wilson, C. M., and Corma, A ., J. Phys. Chem. 97, 13713 (1993).

23. K azansky, V. B., Senchenya, I. N., Frash, M . V., and van Santen, R . A ., Catal. L ett. 27, 345 (1994).

24. Collins, S. J., and O 'M alley, P. J., J. C atal. 153, 94 (1995).

25. Collins, S. J., and O 'M alley, P. J., Chem. Phys. L ett. 246 (1995). 
26. B laszkowski, S. R., Nascimento, M. A. C., and van Santen, R. A., J. Phys. Chem. 100, 3463 (1996).

27. Blaszkowski, S. R ., Jansen, A. P. J., Nascimento, M. A . C., and van Santen, R . A ., J. P hys. Chem. 98, 12938 (1994).

28. K azansky, V. B., Frash, M. V., and van Santen, R. A ., Catal. L ett. 28, 211 (1994).

29. B laszkowski, S. R ., and van Santen, R. A ., J. P hys. Chem. 99, 11728 (1995).

30. Zicovich-Wilson, C. M., Viruela-M artin, P., and Corma, A ., J. Phys. Chem. 99, 13224 (1995).
31. Sie, T., Ind. Chem. Res. 31, 1881 (1992).

32. N owak, A. K., M esters, C. M. A. M., Rigby, A. M., and Schulze, D., manuscript in preparation.

33. Corma, A., M iguel, P. J., and Orchilles, A. V., J. Catal. 145, 171 (1994).

34. H ufton, J. R ., and D anner, R. P., A IChE J. 39, 954 (1993).

35. N arbeshuber, T. F., Vinek, H., and Lercher, J. A ., J. Catal. 157, 388 (1995).

36. Kramer, G. J., and van Santen, R. A ., J. A m. Chem. Soc. 115, 2887 (1993). 